\section{OPEN ACCESS}

Edited by:

Jos J. De Koning,

Vrije Universiteit Amsterdam,

Netherlands

Reviewed by:

Jesús G. Pallarés,

University of Murcia, Spain

Danilo lannetta,

University of Calgary, Canada

*Correspondence:

Almudena Montalvo-Pérez

almudena.montalvo@

universidadeuropea.es

tThese authors have contributed equally to this work

Specialty section: This article was submitted to

Exercise Physiology, a section of the journal

Frontiers in Physiology

Received: 05 March 2021

Accepted: 12 May 2021

Published: 09 June 2021

Citation:

Valenzuela PL, Alejo LB Montalvo-Pérez A, Gil-Cabrera J,

Talavera E, Lucia A and Barranco-Gil D (2021) Relationship Between Critical Power and Different

Lactate Threshold Markers in Recreational Cyclists.

Front. Physiol. 12:676484. doi: 10.3389/fphys.2021.676484

\title{
Relationship Between Critical Power and Different Lactate Threshold Markers in Recreational Cyclists
}

\section{Pedro L. Valenzuela ${ }^{1,2 \dagger}$, Lidia B. Alejo ${ }^{1,2 \dagger}$, Almudena Montalvo-Pérez ${ }^{1 *}$, Jaime Gil-Cabrera ${ }^{1}$, Eduardo Talavera', Alejandro Lucia ${ }^{1,2}$ and David Barranco-Gil'}

${ }^{1}$ Faculty of Sport Sciences, Universidad Europea de Madrid, Madrid, Spain, ${ }^{2}$ Physical Activity and Health Laboratory, Instituto de Investigación Sanitaria Hospital '12 de Octubre' ('imas12'), Madrid, Spain

Purpose: To analyze the relationship between critical power (CP) and different lactate threshold (LT2) markers in cyclists.

Methods: Seventeen male recreational cyclists [33 \pm 5 years, peak power output $(\mathrm{PO})=4.5 \pm 0.7 \mathrm{~W} / \mathrm{kg}$ ] were included in the study. The $\mathrm{PO}$ associated with four different fixed (onset of blood lactate accumulation) and individualized (Dmax $\exp _{\text {, }}$ Dmax pol $_{\text {, and }} \mathrm{LT}_{\Delta 1}$ ) LT2 markers was determined during a maximal incremental cycling test, and CP was calculated from three trials of 1-, 5-, and 20-min duration. The relationship and agreement between each LT2 marker and CP were then analyzed.

Results: Strong correlations ( $r=0.81-0.98$ for all markers) and trivial-to-small non-significant differences (Hedges' $g=0.01-0.17$, bias $=1-9 \mathrm{~W}$, and $p>0.05$ ) were found between all LT2 markers and CP with the exception of $\mathrm{Dmax}_{\text {exp }}$, which showed the strongest correlation but was slightly higher than the CP (Hedges' $g=0.43$, bias $=20 \mathrm{~W}$, and $p<0.001$ ). Wide limits of agreement (LOA) were, however, found for all LT2 markers compared with CP (from $\pm 22 \mathrm{~W}$ for $\operatorname{Dmax}_{\text {exp }}$ to $\pm 52 \mathrm{~W}$ for $\operatorname{Dmax}_{\mathrm{po}}$ ), and unclear to most likely practically meaningful differences ( $P O$ differences between markers $>1 \%$, albeit $<5 \%$ ) were found between markers attending to magnitude-based inferences.

Conclusion: LT2 markers show a strong association and overall trivial-to-small differences with CP. Nevertheless, given the wide LoA and the likelihood of potentially meaningful differences between these endurance-related markers, caution should be employed when using them interchangeably.

Keywords: endurance performance, cycling, peak power output, Dmax method, incremental test

\section{INTRODUCTION}

The workload associated with the transition from steady to non-steady state oxidative metabolism (or heavy- to severe-intensity exercise) has been proposed as one of the main determinants of endurance performance (Poole et al., 2016). Knowledge of this workload might also be useful for training prescription given that training below this boundary level will induce different physiological responses and adaptations than intensities above it (Poole et al., 2016). However, numerous intensity 
markers or "thresholds" have been considered to describe this transition (Jones et al., 2019). For instance, the critical power (CP), the maximal lactate steady state (MLSS), or the respiratory compensation point (RCP) is among the most popular indicators of this threshold (Poole et al., 1988; Iannetta et al., 2020b).

The lactate threshold (LT2) determined during the incremental exercise in the laboratory setting is one of the most traditionally used markers for the assessment of endurance performance and the guidance of training intensity prescription (Joyner and Coyle, 2008; Faude et al., 2009; Iannetta et al., 2020b; Poole et al., 2021). The LT2 is typically defined as the maximum workload that precedes a rapid increase in blood lactate values resulting from an imbalance between lactate production and clearance, which is accompanied by a rise in blood $\mathrm{H}^{+}$(Binder et al., 2008). It should be noted, however, that the workload at which blood lactate demonstrates an accelerated increase can be identified using a variety of methods (Faude et al., 2009). Moreover, while the lactate response to exercise might be reproducible under standardized conditions, physiological factors, such as muscle glycogen concentration or acid-base balance, and methodological factors, such as the testing protocol, can alter the workload at which the LT2 occurs (Jacobs, 1986; Faude et al., 2009; Jamnick et al., 2018).

Under this context, it has been proposed that CP should be considered the "gold standard" index of the transition between steady and non-steady state oxidative metabolism, being a stronger correlate of physical performance than the LT2 (Jones et al., 2019; Poole et al., 2021). Although different mathematical models exist, CP - usually determined through several constant-load tests to exhaustion or time trials of fixed duration - is based on the hyperbolic relationship between external workload [which in the sport of cycling is normally expressed as power output (PO)] and time to fatigue, in which $\mathrm{CP}$ (in watts) is the asymptote of this relationship and $\mathrm{W}^{\prime}$ (in joules) is the curvature constant (Monod and Scherrer, 1965). As with the LT2, CP is also related to endurance performance (Kolbe et al., 1995; Smith et al., 1999), although the CP concept is unique with regard to physiological "thresholds" (e.g., LT2 and RCP) in that its definition is based purely on the measurement of mechanical work done (Jones et al., 2019).

CP and LT2 seem, therefore, to represent similar theoretical concepts, and both are widely used among researchers and coaches for training control and performance assessment. Nevertheless, controversy exists on whether LT2 markers can be used as surrogates of CP (McLellan and Cheung, 1992; Clingeleffer et al., 1994; Smith and Jones, 2001; Dekerle et al., 2003). Accordingly, the aim of the present study was to analyze the relationship between different LT2 markers and CP in recreational cyclists.

\section{MATERIALS AND METHODS}

\section{Participants}

Seventeen male recreational cyclists (age $=33 \pm 5$ years, body mass $=72 \pm 6 \mathrm{~kg}$, and height $=178 \pm 5 \mathrm{~cm}$ ) participated in the study. Inclusion criteria included cycling a minimum of $4 \mathrm{~h}$ per week and a cycling experience greater than 2 years.
Participants were instructed to maintain their normal dietary pattern for the duration of the study and to refrain from performing intense exercise and consuming ergogenic aids, such as caffeine $48 \mathrm{~h}$ prior to each testing session. In agreement with the Committee of Ethics of the University of Alcalá (CEI/HU/2017/26), all participants signed an informed consent form after having the procedures verbally explained.

\section{Experimental Design}

Participants visited the laboratory on two different occasions interspersed by a minimum of $48 \mathrm{~h}$, completing all procedures in a maximum of 1 week. All tests were performed on the same validated indoor cycle trainer (CycleOps, Madison, WI; Lillo-Bevia and Pallarés, 2018), allowing participants to use their own bicycle. During the first session, participants performed a maximal incremental test to characterize the blood lactate curve, which was then modeled using different approaches (Machado et al., 2011, 2012; Gavin et al., 2012; Santos-Concejero et al., 2014). In the second session, participants performed three-time trials of different duration for the computation of CP. All sessions included an initial warm-up consisting of light cycling $(\sim 100 \mathrm{~W})$ for $10 \mathrm{~min}$ at a self-selected cadence.

\section{Lactate Threshold Determination}

Participants performed a maximal incremental cycling test with an initial workload of $150 \mathrm{~W}$, which increased by $25 \mathrm{~W}$ every 3 min until volitional exhaustion or when pedaling cadence was less than $60 \mathrm{rpm}$. This incremental protocol was designed attending to the recommendations by Bentley and co-workers (Bentley et al., 2007), who concluded that 3-min stages represent the optimal option for measuring both maximal and submaximal physiological values. The PPO was determined as the highest, fully completed stage during the incremental maximal test. If the last stage was not fully completed, PPO was calculated according to the formula proposed elsewhere (De Pauw et al., 2013).

Before and at the end of the incremental test as well as after each completed 3-min stage, $0.5 \mu$ l capillary blood samples were drawn from the ear lobe for lactate analysis (Lactate Scout, SensLab GmbH, Germany). The following four LT2 markers were determined with the Lactate-E software

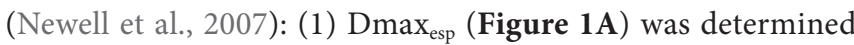
from the lactate-power data fitted by an exponential plus constant regression curve (Machado et al., 2011), corresponding to the point on the regression curve that yielded the maximal perpendicular distance to the straight line connecting the first and last point of the curve; (2) $D_{m_{a x}}$ (Figure 1B) was determined as described for $\operatorname{Dmax}_{\exp }$, with the difference that a third-order polynomial regression curve was used to fit the lactate and power data instead of an exponential plus constant regression curve (Machado et al., 2012; SantosConcejero et al., 2014); (3) The onset of blood lactate accumulation (OBLA; Figure 1C), which corresponded to the power equivalent to a blood lactate concentration of $4 \mathrm{mmol} / \mathrm{l}$ determined by interpolation from a third-order polynomial regression model of the lactate vs. work load 
A

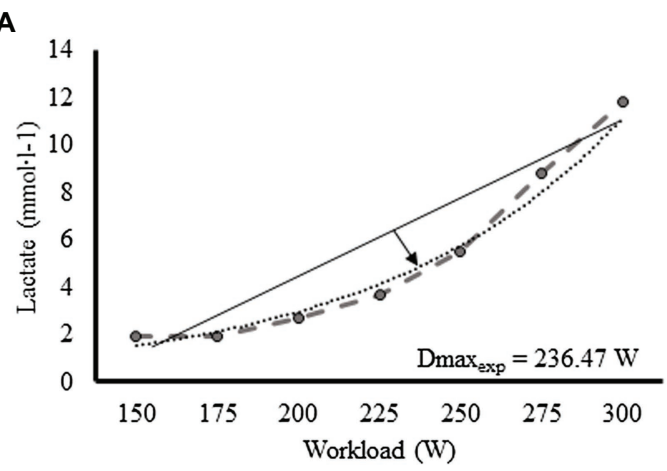

C

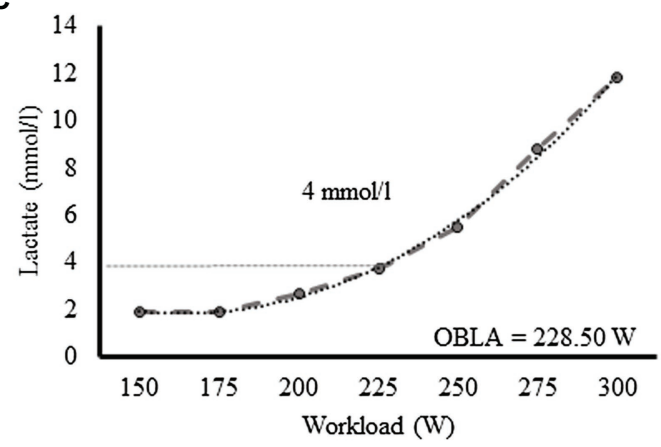

B

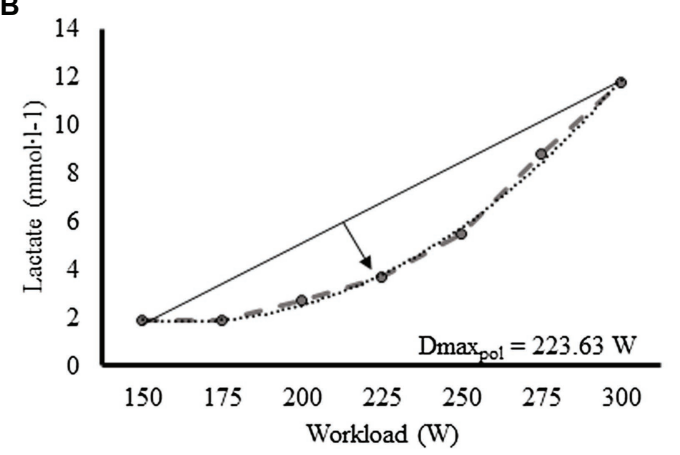

D

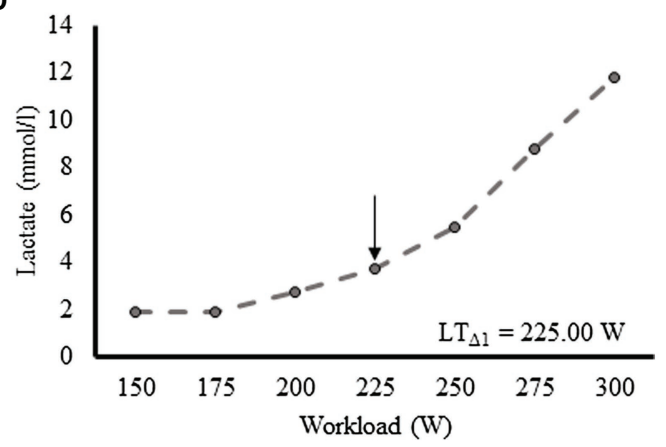

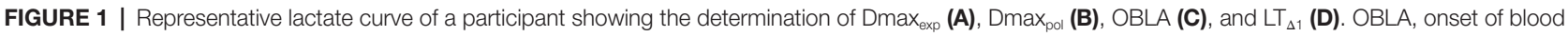
lactate accumulation.

curve; and (4) $\mathrm{LT}_{\Delta 1}$ (Figure 1D) considered as the point at which blood lactate values increased $\geq 1 \mathrm{mmol} / \mathrm{l}$ with regard to the previous stage (Gavin et al., 2012). These LT2 markers have been widely used in the scientific literature and proven to be related to endurance performance (Gavin et al., 2012; Machado et al., 2012; Santos-Concejero et al., 2014).

\section{Critical Power Determination}

Participants performed three-time trials of 1, 5, and $20 \mathrm{~min}-$ always in this order - and the mean PO was registered. The three trials were performed in the same session and were interspersed by $30 \mathrm{~min}$ of passive recovery, as previously reported (Galbraith et al., 2014; Triska et al., 2015; Karsten et al., 2021). No specific pacing strategy was recommended, although participants were encouraged to achieve the highest mean PO possible during the trials. Participants were allowed to change gears during the test so as to maintain their preferred cadence.

Critical power was determined as the slope of the regression line of work against time following the equation (Moritani et al., 1981):

$$
\text { Work }(\text { Jules })=W^{\prime}(\text { Jules })+C P(\text { Watts }) \cdot \text { Duration }(\mathrm{sec})
$$

\section{Statistical Analysis}

Normality (Kolmogorov-Smirnov test) and homoscedasticity (Levene's test) of the data were checked prior to any statistical treatment. Differences between each LT2 marker and CP were determined using Student's paired $t$-tests. The magnitude of the differences (effect size) was assessed using Hedges' $g$ and were considered trivial $(g<0.20)$, small $(g<0.60)$, moderate $(g<1.20)$, large $(g<2.00)$, or very large $(g>2.00$; Hopkins et al., 2009). The bias and limits of agreement (LoA) between markers were assessed using Bland-Altman plots. Differences between markers were also assessed through magnitude-based inferences with a $90 \%$ confidence level setting two different smallest worthwhile changes (SWC; Batterham and Hopkins, 2006; Karsten et al., 2021). An SWC of 1\% was chosen because this value has been reported to represent a meaningful change in performance in cyclists (i.e., above the random error of measurement and also above the typical day-to-day variation in performance; Paton and Hopkins, 2001, 2006; Hopkins, 2004). An SWC of 5\% was also analyzed given that variations in $\mathrm{PO}$ of this magnitude have been reported to suffice for modifying the steady physiological response to exercise at $\mathrm{CP}$ (Poole et al., 1988). Pearson's correlation analysis and Lin's concordant coefficient were used to examine the relationship between markers. Values of $r$ of $0.1,0.3,0.5,0.7$, and 0.9 were considered small, moderate, large, very large, and extremely large, respectively (Hopkins et al., 2009). The standard error of estimate was used to examine the relationship between the different markers. Data are shown as mean \pm SD. Statistical analyses were performed with a spreadsheet (Hopkins, 2015) and statistical software (SPSS 26.0, Inc., Chicago, IL), setting the alpha for significance at 0.05 . 


\section{RESULTS}

Participants' PPO was $321 \pm 40 \mathrm{~W}$ (or $4.5 \pm 0.7 \mathrm{~W} / \mathrm{kg}$ ). The mean PO associated with the $\operatorname{Dmax}_{\text {exp }}, \operatorname{Dmax}_{\mathrm{pol}}, \mathrm{OBLA}$, and $\mathrm{LT}_{\Delta 1}$ was $273 \pm 49(84.6 \pm 6.0 \%$ of PPO), $252 \pm 42(78.6 \pm 9.4 \%$ of PPO), $261 \pm 53(80.7 \pm 8.7 \%$ of PPO), and $247 \pm 43 \mathrm{~W}$ (76.5 $\pm 7.2 \%$ of PPO), respectively. Participants' mean PO during the 1-, 5-, and 20-min time trials was $477 \pm 72$, $318 \pm 55$, and $265 \pm 45 \mathrm{~W}$, respectively. The resultant $\mathrm{CP}$ was $252 \pm 44 \mathrm{~W}(78.3 \pm 6.2 \%$ of PPO $)$.

All LT2 markers were strongly correlated with each other (Values of $r$ ranging from 0.73 to 0.95 ), although small but significant differences were found between $D_{\max }$ exp and the other LT2 markers examined (bias ranging from 9 to $18 \mathrm{~W}$ ), as well as between OBLA and $\mathrm{LT}_{\Delta 1}$ (bias = $11 \mathrm{~W}$; Table 1).

The relationship and agreement of each LT2 marker with CP are shown in Figures 2, 3, respectively. CP was significantly and strongly correlated with all LT2 markers (Values of $r$

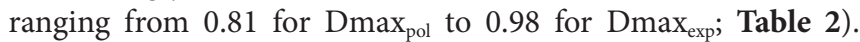
Non-significant and trivial-to-small differences were found between all LT2 markers and CP (bias ranging from 1 to 9 W) except for $D_{\text {max }}$ exp, which was significantly and largely higher than CP (bias $=20 \mathrm{~W}$; Table 2). Wide LoA were, however, found for all LT2 markers when compared with CP, which ranged between $\pm 22 \mathrm{~W}$ for $\mathrm{Dmax}_{\exp }$ and $\pm 52 \mathrm{~W}$ for $\operatorname{Dmax}_{\text {pol }}$ (Figure 3; Table 2). The likelihood of differences $>1 \%$ between CP and LT2 markers was 100\% (most likely), 33\% (unclear), 82\% (likely), and 70\% (unclear) for Dmax $\exp _{\text {ex }}, \mathrm{Dmax}_{\mathrm{pol}}$, OBLA, and $\mathrm{LT}_{\Delta 1}$, respectively, whereas the likelihood of differences $>5 \%$ with CP was $2 \%$ (unclear), $0 \%$ (unclear), $0 \%$ (unclear), and $0 \%$ (unclear), respectively.

\section{DISCUSSION}

In the present study, we found that LT2 markers - including the fixed marker OBLA and the individualized markers Dmax ${ }_{\text {exp }}$, $\mathrm{Dmax}_{\mathrm{pol}}$, or $\mathrm{LT}_{\Delta 1}$ - are strongly correlated with $\mathrm{CP}$ in recreational cyclists. Also, no significant differences were observed between the LT2 markers and CP, with the exception of Dmax exp. However, the wide LoA and the likelihood of practically meaningful differences $(>1 \%$, which would be of relevance for performance purposes) found between these indices raise concern on whether they could be used interchangeably.

Different markers associated with the so-called "anaerobic threshold" have been compared with CP in the scientific literature, and mixed results have been reported for their association (Galán-Rioja et al., 2020). In a recent metaanalysis, CP was reported to be significantly correlated with different indices, such as the first and second ventilatory threshold (the latter also known as "RCP") or the MLSS, but the LT2 was not assessed (Galán-Rioja et al., 2020). Regarding the latter, CP (or "critical velocity" for running exercise) has been reported to be significantly correlated with the individual anaerobic threshold (McLellan and Cheung, 1992) and with the OBLA (Housh et al., 1991; Denadai et al., 2005), although controversy exists over its association with the LT2 determined by visual inspection (Smith and Jones, 2001). Our study suggests that most LT2 markers - at least those assessed here - are associated with CP, although the strength of this association might partially depend on the analyzed marker, with $\operatorname{Dmax}_{\exp }$ emerging as the most strongly correlated $(r=0.98)$. Given that $\mathrm{CP}$ has been proposed as the gold standard index of the heavy- to severe-intensity exercise transition (Jones et al., 2019; Poole et al., 2021) and has proven to be strongly related to endurance performance (Kolbe et al., 1995; Smith et al., 1999), our findings support the assessment of LT2 markers and, particularly, $\operatorname{Dmax}_{\exp }$ as an alternative method to estimate CP and predict endurance performance. Indeed, the previous studies have shown the superiority of $\operatorname{Dmax}_{\exp }$ over other LT2 markers (including OBLA, $\operatorname{Dmax}_{\text {pol }}$, or $\mathrm{LT}_{\Delta 1}$ ) for the prediction of endurance performance (Nicholson and Sleivert, 2001; Machado et al., 2011, 2012; Santos-Concejero et al., 2014).

Despite their potential association, mixed evidence exists regarding the agreement of equivalence between LT2 markers and CP. For instance, CP (or critical speed) has been reported to be higher than the individual anaerobic threshold (McLellan and Cheung, 1992) and that the OBLA (Housh et al., 1991; Clingeleffer et al., 1994) in some studies, but others reported no significant differences between CP and the LT2 determined

TABLE 1 | Relationship between the power output associated with different lactate threshold markers

\begin{tabular}{|c|c|c|c|c|c|c|c|}
\hline & \multicolumn{3}{|c|}{ Relationship } & \multicolumn{4}{|c|}{ Agreement } \\
\hline & $r$ & $p$ & SEE (W) & LoA (W) & CCC & $\begin{array}{l}\text { Student's } t \text {-test } \\
\text { Value of } p\end{array}$ & Hedges' $g$ \\
\hline Dmax $_{\text {exp }}-$ Dmax $_{\text {pol }}$ & 0.73 & $<0.001$ & 23.6 & $18.4 \pm 33.5$ & 0.659 & 0.020 & 0.45 \\
\hline Dmax $_{\text {exp }}-O B L A$ & 0.95 & $<0.001$ & 11.2 & $8.7 \pm 15.8$ & 0.926 & 0.008 & 0.22 \\
\hline $\mathrm{Dmax}_{\mathrm{exp}}-\mathrm{LT}_{\Delta 1}$ & 0.84 & $<0.001$ & 18.4 & $14.3 \pm 26.0$ & 0.717 & $<0.001$ & 0.56 \\
\hline Dmax $_{\text {pol }}-O B L A$ & 0.82 & $<0.001$ & 21.2 & $16.5 \pm 30.1$ & 0.791 & 0.223 & 0.19 \\
\hline $\operatorname{Dmax}_{\text {pol }}-\mathrm{LT}_{\Delta 1}$ & 0.76 & $<0.001$ & 21.0 & $16.4 \pm 29.8$ & 0.754 & 0.474 & 0.12 \\
\hline OBLA-LT $T_{\Delta 1}$ & 0.93 & $<0.001$ & 13.9 & $10.8 \pm 19.7$ & 0.877 & 0.008 & 0.29 \\
\hline
\end{tabular}

CCC, Lin's concordant coefficient; OBLA, onset of blood lactate accumulation; LOA, limit of agreement presented as bias $\pm 1.96 *$ standard deviation; and SEE, standard estimated error. 

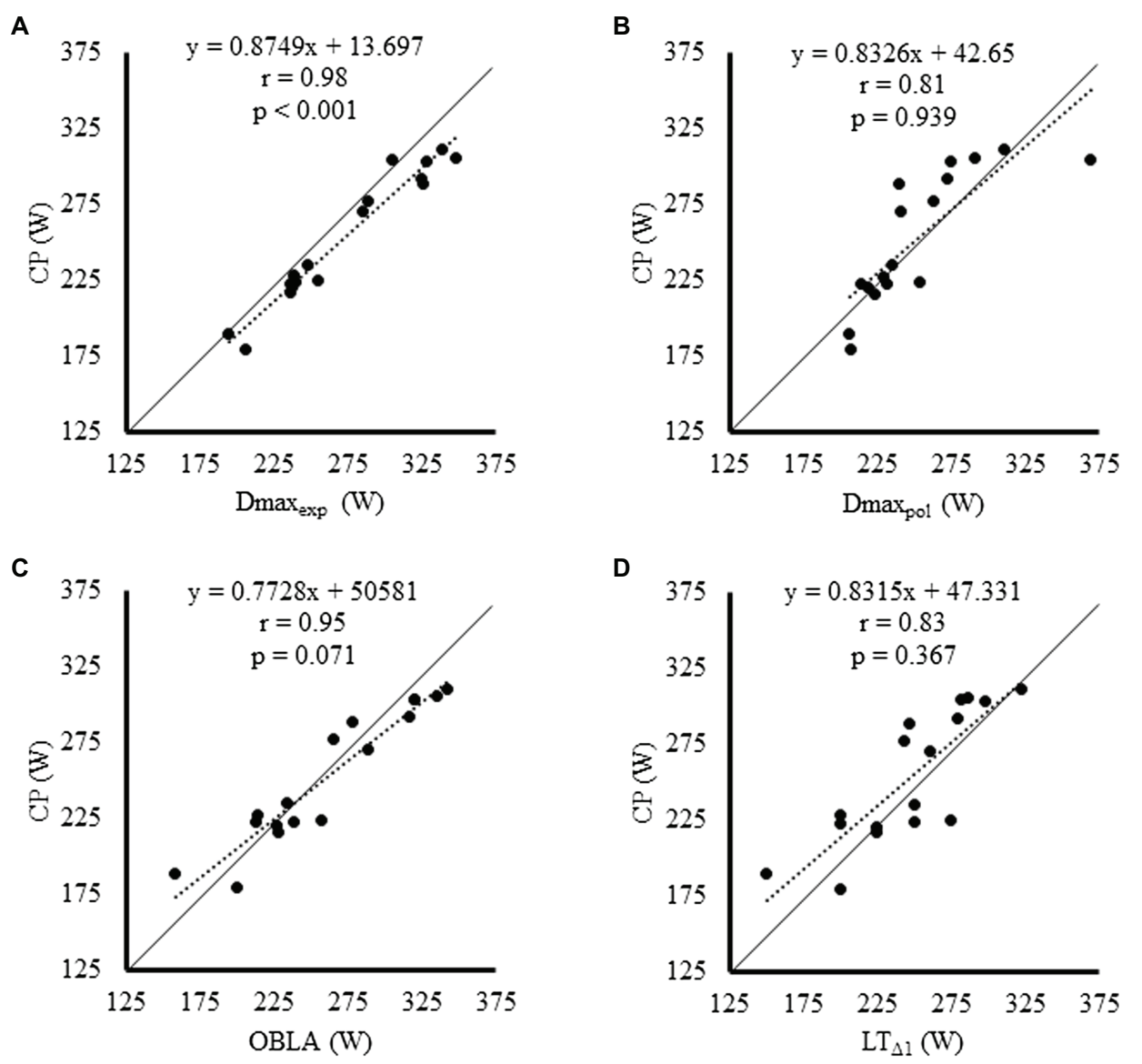

FIGURE 2 | Relationship between each lactate threshold marker [Dmax $\exp (\mathbf{A})$, Dmax $_{\text {pol }}$ (B), OBLA (C), and LT (D) $_{\text {(D) }}$ and critical power. OBLA, onset of blood lactate accumulation.

by visual inspection (Smith and Jones, 2001) or between CP and OBLA (determined as a blood lactate concentration of $3.5 \mathrm{mmol} / \mathrm{l}$ instead of $4.0 \mathrm{mmol} / \mathrm{l}$; Denadai et al., 2005). Our results indicate that, besides being strongly correlated, CP and LT2 markers showed overall trivial-to-small differences. Nevertheless, despite the strong correlations and the absence of significant differences, wide LoA [from $\pm 22 \mathrm{~W}(\sim 10 \%)$ to $\pm 52 \mathrm{~W}(\sim 20 \%)]$ and some likelihood $(33-100 \%)$ of differences that might be practically relevant for performance purposes (i.e., $>1 \%$, which is above the random error for the variability of cyclists' performance between days) were found between markers. It must be noted, nonetheless, that the likelihood of differences between markers $>5 \%$ was unclear. Differences $>5 \%$ might have real-world relevance for training purposes. Although exercise at CP may not always elicit steady-state physiological responses (Overend et al., 1992; Brickley et al., 2002; Pringle and Jones, 2002; Sawyer et al., 2012; Mattioni Maturana et al., 2016), it is accepted that exercise below vs. above $(\sim+5 \%)$ this threshold does differentiate between heavy and severe exercise on the basis of several physiological profiles (e.g., oxygen uptake, lactate, and pH; Poole et al., 1988; Jones et al., 2008). Similarly, there is disagreement on whether exercising at the LT2 elicits a steady physiological response, which might partly depend on the LT2 marker analyzed (Schnabel et al., 1982; Stegmann and Kindermann, 1982; Ribeiro et al., 1986; Oyono-Enguelle et al., 1990; Urhausen, 1993; Baldari and Guidetti, 2000; Lajoie et al., 2000; Jamnick et al., 2018) as well as on the protocol used for LT2 determination (Simon et al., 1983). Thus, the present findings suggest that LT2 markers are unlikely to overestimate or underestimate $\mathrm{CP}$ by more than $5 \%$. However, given that small variations in exercise intensity (probably $<5 \%$ ) can result in different physiological responses and adaptations, caution should be taken when indistinctly using these markers for training prescription (Granata et al., 2018; Iannetta et al., 2020a).

Some methodological considerations of the present study deserve comment. We aimed to compare an "effort-dependent" marker obtained during several time trials, CP, with an "effort-independent" marker obtained during incremental exercise, the LT2. Effort-independent markers of physiologic thresholds have been reported to have some benefits over effort-independent markers because they do not rely on participants' volition or perceptions, being preferable for testing vulnerable populations (e.g., patients or very old people; Poole et al., 2021). However, the LT2 determination can be affected by several variables including the specific 
A

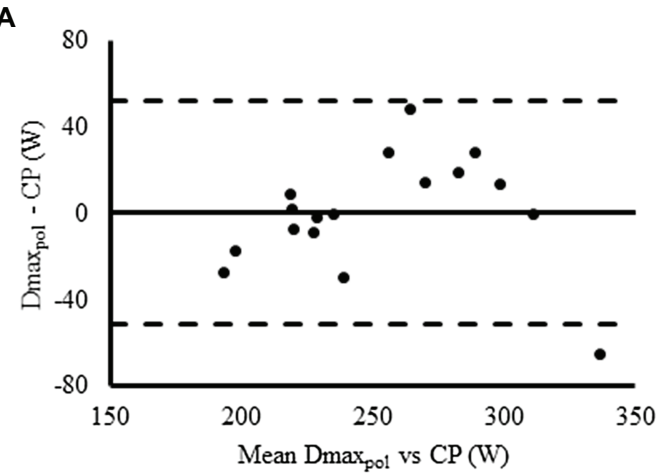

C

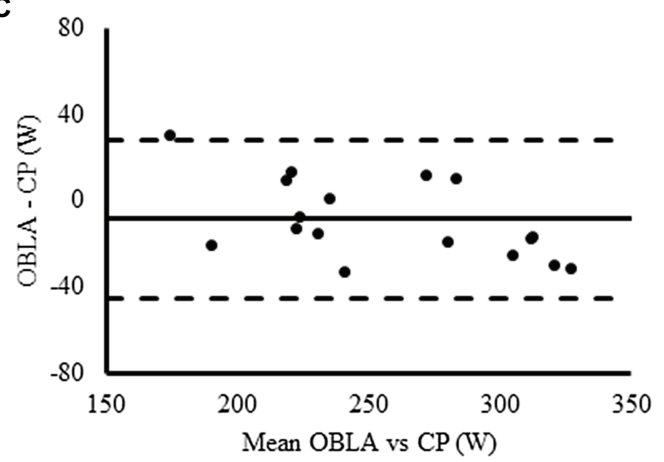

B

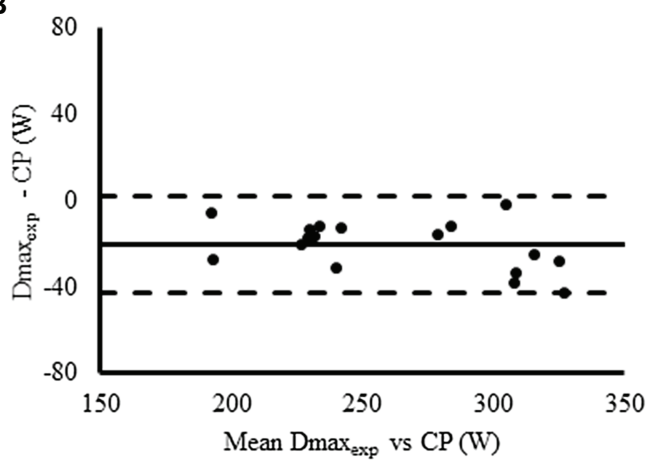

D

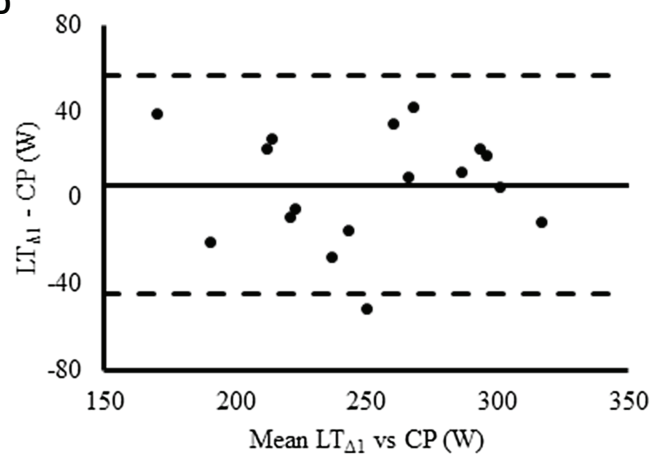

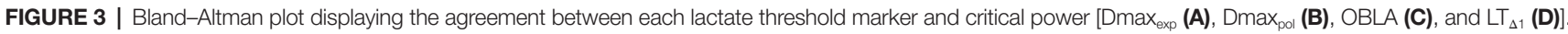
Solid and dashed horizontal lines represent the bias and the limits of agreement (bias $\pm 1.96 \mathrm{SD}$ ), respectively.

TABLE 2 | Relationship and agreement between the power output corresponding to different lactate threshold markers and critical power.

\begin{tabular}{|c|c|c|c|c|c|c|c|}
\hline & \multicolumn{3}{|c|}{ Relationship with CP } & \multicolumn{4}{|c|}{ Agreement with CP } \\
\hline & $r$ & $p$ & SEE (W) & LoA (W) & $\operatorname{ccc}$ & $\begin{array}{l}\text { Student's } t \text {-test } \\
\text { Value of } p\end{array}$ & Hedges' $g$ \\
\hline $\operatorname{Dmax}_{\text {pol }}$ & 0.81 & $<0.001$ & 26.4 & $0.5 \pm 51.9$ & 0.810 & 0.939 & 0.01 \\
\hline $\operatorname{Dmax}_{\exp }$ & 0.98 & $<0.001$ & 9.9 & $-20.4 \pm 22.3$ & 0.878 & $<0.001$ & 0.43 \\
\hline OBLA & 0.95 & $<0.001$ & 14.6 & $5.8 \pm 50.4$ & 0.912 & 0.071 & 0.17 \\
\hline
\end{tabular}

CP, critical power; CCC, Lin's concordant coefficient; LOA, limit of agreement presented as bias \pm 1.96 " standard deviation; OBLA, onset of blood lactate accumulation; and SEE, standard estimated error.

marker analyzed, glycogen store status, acid-base balance, or testing protocol (e.g., stage duration; Jacobs, 1986; Faude et al., 2009; Jamnick et al., 2018; Poole et al., 2021). For this reason, it has been proposed that when individuals are able to undertake high-intensity exhausting exercise, CP offers the greatest potential to predict athletic performance, assess physiological function, and monitor training efficacy (Poole et al., 2021). Moreover, as previously reported for gas exchange thresholds (e.g., RCP), the comparison of physiological markers, such as LT2 obtained during incremental exercise with others assessed during constant exercise (i.e., CP), might induce some bias due to an overestimation of the former (Iannetta et al., 2019; Caen et al., 2021), although an incremental test with 3-min stages, such as the one used in the present study, is expected to be sufficient to elicit a steady physiological state (Bentley et al., 2007). On the other hand, different LT2 markers have been used in the scientific literature (Faude et al., 2009), but here, we just analyzed some of the most popular ones and therefore, different LT2 markers might yield different results. The protocol used for the determination of CP can also affect its magnitude (Triska et al., 2018). In this regard, following the protocol used in the previous studies (Galbraith et al., 2014; Triska et al., 2015; Karsten et al., 2021), we conducted a single-day protocol for the assessment of CP with a 30-min rest between time trials, which has been reported to provide similar $\mathrm{CP}$ estimates compared with other protocols with a longer rest period (Galbraith et al., 2014). However, it might be hypothesized that fatigue could potentially impair the PO attained during 
the time trials and consequently the estimation of CP. We are unable to ascertain whether this was actually the case in the present study as no physiological verifications of the $\mathrm{CP}$ estimates were performed. Therefore, our findings should be confirmed using different protocols.

In summary, the present study indicates a strong association between different LT2 markers and CP in recreational cyclists. However, although differences between LT2 markers and CP were overall trivial-to-small, the wide LoA found and the likelihood of practically meaningful differences (albeit lower than $5 \%$ ) raise concerns on the suitability of using these markers interchangeably for training prescription, as they might potentially induce different physiological responses and adaptations.

\section{DATA AVAILABILITY STATEMENT}

The original contributions presented in the study are included in the article/supplementary material, and further inquiries can be directed to the corresponding author.

\section{ETHICS STATEMENT}

The studies involving human participants were reviewed and approved by the Committee of Ethics of the University of

\section{REFERENCES}

Baldari, C., and Guidetti, L. (2000). A simple method for individual anaerobic threshold as predictor of max lactate steady state. Med. Sci. Sports Exerc. 32, 1798-1802. doi: 10.1097/00005768-200010000-00022

Batterham, A. M., and Hopkins, W. G. (2006). Making meaningful inferences about magnitudes. Int. J. Sports Physiol. Perform. 1, 50-57. doi: 10.1123/ijspp.1.1.50

Bentley, D. J., Newell, J., and Bishop, D. (2007). Incremental exercise test design and analysis: implications for performance diagnostics in endurance athletes. Sports Med. 37, 575-586. doi: 10.2165/00007256-200737070-00002

Binder, R. K., Wonisch, M., Corra, U., Cohen-Solal, A., Vanhees, L., Saner, H., et al. (2008). Methodological approach to the first and second lactate threshold in incremental cardiopulmonary exercise testing. Eur. J. Cardiovasc. Prev. Rehabil. 15, 726-734. doi: 10.1097/HJR.0b013e328304fed 4

Brickley, G., Doust, J., and Williams, C. A. (2002). Physiological responses during exercise to exhaustion at critical power. Eur. J. Appl. Physiol. 88, 146-151. doi: 10.1007/s00421-002-0706-1

Caen, K., Pogliaghi, S., Lievens, M., Vermeire, K., Bourgois, J. G., and Boone, J. (2021). Ramp vs. step tests: valid alternatives to determine the maximal lactate steady-state intensity? Eur. J. Appl. Physiol. 1-9. doi: 10.1007/s00421-021-04620-9

Clingeleffer, A., Mc Naughton, L. R., and Davoren, B. (1994). The use of critical power as a determinant for establishing the onset of blood lactate accumulation. Eur. J. Appl. Physiol. Occup. Physiol. 68, 182-187. doi: 10.1007/ BF00244033

De Pauw, K., Roelands, B., Cheung, S. S., De Geus, B., Rietjens, G., and Meeusen, R. (2013). Guidelines to classify subject groups in sport-science research. Int. J. Sports Physiol. Perform. 8, 111-122. doi: 10.1123/ijspp.8.2.111

Dekerle, J., Baron, B., Dupont, L., Vanvelcenaher, J., and Pelayo, P. (2003). Maximal lactate steady state, respiratory compensation threshold and critical power. Eur. J. Appl. Physiol. 89, 281-288. doi: 10.1007/s00421-002-0786-y

Denadai, B. S., Gomide, E. B. G., and Greco, C. C. (2005). The relationship between onset of blood lactate accumulation, critical velocity, and maximal lactate steady state in soccer players. J. Strength Cond. Res. 19, 364-368. doi: 10.1519/00124278-200505000-00021
Alcalá (CEI/HU/2017/26). The patients/participants provided their written informed consent to participate in this study.

\section{AUTHOR CONTRIBUTIONS}

Conception and design of the experiments and drafting of the manuscript: PV. Experimental preparation and data collection: PV, DB-G, LA, AM-P, JG-C, and ET. Analysis and interpretation: PV, DB-G, LA, AM-P, JG-C, ET, and AL. Revision of the manuscript for important intellectual content: All authors. All authors contributed to the article and approved the submitted version.

\section{FUNDING}

Research by AL is funded by the Spanish Ministry of Economy and Competitiveness and Fondos Feder (AL, grant PI18/00139).

\section{ACKNOWLEDGMENTS}

We thank all the participants for their help and cooperation during the study.

Faude, O., Kindermann, W., and Meyer, T. (2009). Lactate threshold concepts: how valid are they? Sports Med. 39, 469-490. doi: 10.2165/00007256-2009 39060-00003

Galán-Rioja, M. Á., González-Mohíno, F., Poole, D. C., and González-Ravé, J. M. (2020). Relative proximity of critical power and metabolic/ventilatory thresholds: systematic review and meta-analysis. Sports Med. 50, 1771-1783. doi: 10.1007/ s40279-020-01314-8

Galbraith, A., Hopker, J., Lelliott, S., Diddams, L., and Passfield, L. (2014). A single-visit field test of critical speed. Int. J. Sports Physiol. Perform. 9, 931-935. doi: 10.1123/ijspp.2013-0507

Gavin, T. P., Van Meter, J. B., Brophy, P. M., Dubis, G. S., Potts, K. N., and Hickner, R. C. (2012). Comparison of a field-based test to estimate functional threshold power and power output at lactate threshold. J. Strength Cond. Res. 26, 416-421. doi: 10.1519/JSC.0b013e318220b4eb

Granata, C., Jamnick, N. A., and Bishop, D. J. (2018). Principles of exercise prescription, and how they influence exercise-induced changes of transcription factors and other regulators of mitochondrial biogenesis. Sports Med. 48, 1541-1559. doi: 10.1007/s40279-018-0894-4

Hopkins, W. (2015). Spreadsheets for analysis of validity and reliability. Sportscience $36-42$.

Hopkins, W. G. (2004). How to interpret changes in an athletic performance test. Sportscience 8, 1-7.

Hopkins, W. G., Marshall, S. W., Batterham, A. M., and Hanin, J. (2009). Progressive statistics for studies in sports medicine and exercise science. Med. Sci. Sports Exerc. 41, 3-13. doi: 10.1249/MSS.0b013e31818cb278

Housh, T. J., Devries, H. A., Housh, D. J., Tichy, M. W., Smyth, K. D., and Tichy, A. M. (1991). The relationship between critical power and the onset of blood lactate accumulation. J. Sports Med. Phys. Fitness 31, 31-36.

Iannetta, D., de Almeida Azevedo, R., Keir, D. A., and Murias, J. M. (2019, 1985). Establishing the Vo2 versus constant-work-rate relationship from ramp-incremental exercise: simple strategies for an unsolved problem. J. Appl. Physiol. 127, 1519-1527. doi: 10.1152/japplphysiol.00508.2019

Iannetta, D., Inglis, E. C., Mattu, A. T., Fontana, F. Y., Pogliaghi, S., Keir, D. A., et al. (2020a). A critical evaluation of current methods for exercise prescription 
in women and men. Med. Sci. Sports Exerc. 52, 466-473. doi: 10.1249/ MSS.0000000000002147

Iannetta, D., Inglis, E. C., Pogliaghi, S., Murias, J. M., and Keir, D. A. (2020b). A "step-ramp-step" protocol to identify the maximal metabolic steady state. Med. Sci. Sports Exerc. 52, 2011-2019. doi: 10.1249/MSS.0000000000002343

Jacobs, I. (1986). Blood lactate. Implications for training and sports performance. Sports Med. 3, 10-25. doi: 10.2165/00007256-198603010-00003

Jamnick, N. A., Botella, J., Pyne, D. B., and Bishop, D. J. (2018). Manipulating graded exercise test variables affects the validity of the lactate threshold and V'O2peak. PLoS One 13:e0199794. doi: 10.1371/journal.pone.0199794

Jones, A. M., Burnley, M., Black, M. I., Poole, D. C., and Vanhatalo, A. (2019). The maximal metabolic steady state: redefining the 'gold standard.' Phys. Rep. 7:e14098. doi: 10.14814/phy2.14098

Jones, A. M., Wilkerson, D. P., DiMenna, F., Fulford, J., and Poole, D. C. (2008). Muscle metabolic responses to exercise above and below the "critical power" assessed using 31P-MRS. Am. J. Phys. Regul. Integr. Comp. Phys. 294, R585-R593. doi: 10.1152/ajpregu.00731.2007

Joyner, M. J., and Coyle, E. F. (2008). Endurance exercise performance: the physiology of champions. J. Physiol. 586, 35-44. doi: 10.1113/ jphysiol.2007.143834

Karsten, B., Petrigna, L., Klose, A., Bianco, A., Townsend, N., and Triska, C. (2021). Relationship between the critical power test and a 20-min functional threshold power test in cycling. Front. Physiol. 11:613151. doi: 10.3389/ fphys.2020.613151

Kolbe, T., Dennis, S. C., Selley, E., Noakes, T. D., and Lambert, M. I. (1995). The relationship between critical power and running performance. J. Sports Sci. 13, 265-269. doi: 10.1080/02640419508732236

Lajoie, C., Laurencelle, L., and Trudeau, F. (2000). Physiological responses to cycling for $60 \mathrm{~min}$ at maximal lactate steady state. J. Chem. Inf. Model. 25, 250-261. doi: 10.1017/CBO9781107415324.004

Lillo-Bevia, J. R., and Pallarés, J. G. (2018). Validity and reliability of the Cycleops hammer cycle ergometer. Int. J. Sports Physiol. Perform. 13, 853-859. doi: 10.1123/ijspp.2017-0403

Machado, F. A., De Moraes, S. M. F., Peserico, C. S., Mezzaroba, P. V., and Higino, W. P. (2011). The Dmax is highly related to performance in middleaged females. Int. J. Sports Med. 32, 672-676. doi: 10.1055/s-0031-1275671

Machado, F. A., Nakamura, F. Y., and Moraes, S. M. F.De. (2012). Influence of regression model and incremental test protocol on the relationship between lactate threshold using the maximal-deviation method and performance in female runners. J. Sports Sci. 30, 1267-1274. doi:10.1080/02640414.2012.702424.

Mattioni Maturana, F., Keir, D. A., McLay, K. M., and Murias, J. M. (2016). Can measures of critical power precisely estimate the maximal metabolic steady-state? Appl. Physiol. Nutr. Metab. 41, 1197-1203. doi: 10.1139/ apnm-2016-0248

McLellan, T. M., and Cheung, K. S. (1992). A comparative evaluation of the individual anaerobic threshold and the critical power. Med. Sci. Sports Exerc. 24, 543-550.

Monod, H., and Scherrer, J. (1965). The work capacity of a synergic muscular group. Ergonomics 8, 329-338. doi: 10.1080/00140136508930810

Moritani, T., Nagata, A., Devries, H. A., and Muro, M. (1981). Critical power as a measure of physical work capacity and anaerobic threshold. Ergonomics 24, 339-350. doi: 10.1080/00140138108924856

Newell, J., Higgins, D., Madden, N., Cruickshank, J., Einbeck, J., McMillan, K., et al. (2007). Software for calculating blood lactate endurance markers. J. Sports Sci. 25, 1403-1409. doi: 10.1080/02640410601128922

Nicholson, R. M., and Sleivert, G. G. (2001). Indices of lactate threshold and their relationship with $10-\mathrm{km}$ running velocity. Med. Sci. Sports Exerc. 33, 339-342. doi: 10.1097/00005768-200102000-00026

Overend, T. J., Cunningham, D. A., Paterson, D. H., and Smith, W. D. (1992). Physiological responses of young and elderly men to prolonged exercise at critical power. Eur. J. Appl. Physiol. Occup. Physiol. 64, 187-193. doi: 10.1007/ BF00717959

Oyono-Enguelle, S., Heitz, A., Marbach, J., Ott, C., Gartner, M., Pape, A., et al. (1990). Blood lactate during constant-load exercise at aerobic and anaerobic thresholds. Eur. J. Appl. Physiol. Occup. Physiol. 60, 321-330. doi: 10.1007/BF00713494
Paton, C., and Hopkins, W. (2006). Variation in performance of elite cyclists from race to race. Eur. J. Sport Sci. 6, 25-31. doi: 10.1080/17461390500422796

Paton, C. D., and Hopkins, W. G. (2001). Tests of cycling performance. Sports Med. 31, 489-496. doi: 10.2165/00007256-200131070-00004

Poole, D. C., Burnley, M., Vanhatalo, A., Rossiter, H. B., and Jones, A. M. (2016). Critical power: an important fatigue threshold in exercise physiology. Med. Sci. Sports Exerc. 48, 2320-2334. doi: 10.1249/MSS.0000000000000939

Poole, D. C., Rossiter, H. B., Brooks, G. A., and Gladden, L. B. (2021). The anaerobic threshold: 50+ years of controversy. J. Physiol. 599, 737-767. doi: 10.1113/JP279963

Poole, D. C., and Ward, S. A., Gardner, G. W., and Whipp, B. J., (1988). Metabolic and respiratory profile of the upper limit for prolonged exercise in man. Ergonomics 31, 1265-1279. doi: 10.1080/00140138808966766

Pringle, J. S. M., and Jones, A. M. (2002). Maximal lactate steady state, critical power and EMG during cycling. Eur. J. Appl. Physiol. 88, 214-226. doi: 10.1007/s00421-002-0703-4

Ribeiro, J. P., Hughes, V., Fielding, R. A., Holden, W., Evans, W., and Knuttgen, H. G. (1986). Metabolic and ventilatory responses to steady state exercise relative to lactate thresholds. Eur. J. Appl. Physiol. Occup. Physiol. 55, 215-221. doi: 10.1007/BF00715008

Santos-Concejero, J., Tucker, R., Granados, C., Irazusta, J., Bidaurrazaga-Letona, I., Zabala-Lili, J., et al. (2014). Influence of regression model and initial intensity of an incremental test on the relationship between the lactate threshold estimated by the maximal-deviation method and running performance. J. Sports Sci. 32, 853-859. doi: 10.1080/02640414.2013.862844

Sawyer, B. J., Morton, R. H., Womack, C. J., and Gaesser, G. A. (2012). V`O2max may not be reached during exercise to exhaustion above critical power. Med. Sci. Sports Exerc. 44, 1533-1538. doi: 10.1249/MSS.0b013e31824d2587

Schnabel, A., Kindermann, W., Schmitt, W. M., Biro, G., and Stegmann, H. (1982). Hormonal and metabolic consequences of prolonged running at the individual anaerobic threshold. Int. J. Sports Med. 3, 163-168. doi: 10.1055/ s-2008-1026082

Simon, J., Young, J. L., Gutin, B., Blood, D. K., and Case, R. B. (1983). Lactate accumulation relative to the anaerobic and respiratory compensation thresholds. J. Appl. Physiol. Respir. Environ. Exerc. Physiol. 54, 13-17. doi: 10.1152/ jappl.1983.54.1.13

Smith, C. G. M., and Jones, A. M. (2001). The relationship between critical velocity, maximal lactate steady-state velocity and lactate turnpoint velocity in runners. Eur. J. Appl. Physiol. 85, 19-26. doi: 10.1007/s004210100384

Smith, J. C., Dangelmaier, B. S., and Hill, D. W. (1999). Critical power is related to cycling time trial performance. Int. J. Sports Med. 20, 374-378. doi: $10.1055 /$ s-2007-971147

Stegmann, H., and Kindermann, W. (1982). Comparison of prolonged exercise tests at the individual anaerobic threshold and the fixed anaerobic threshold of 4 mmoll lactate. Int. J. Sports Med. 3, 105-110. doi: 10.1055/s-2008-1026072

Triska, C., Karsten, B., Beedie, C., Koller-Zeisler, B., Nimmerichter, A., and Tschan, H. (2018). Different durations within the method of best practice affect the parameters of the speed-duration relationship. Eur. J. Sport Sci. 18, 332-340. doi: 10.1080/17461391.2017.1418025

Triska, C., Tschan, H., Tazreiter, G., and Nimmerichter, A. (2015). Critical power in laboratory and field conditions using single-visit maximal effort trials. Int. J. Sports Med. 36, 1063-1068. doi: 10.1055/s-0035-1549958

Urhausen, A. (1993). Individual anaerobic threshold and maximum lactate steady state. Int. J. Sports Med. 14, 134-139. doi: 10.1055/s-2007-1021157

Conflict of Interest: The authors declare that the research was conducted in the absence of any commercial or financial relationships that could be construed as a potential conflict of interest.

Copyright (c) 2021 Valenzuela, Alejo, Montalvo-Pérez, Gil-Cabrera, Talavera, Lucia and Barranco-Gil. This is an open-access article distributed under the terms of the Creative Commons Attribution License (CC BY). The use, distribution or reproduction in other forums is permitted, provided the original author(s) and the copyright owner(s) are credited and that the original publication in this journal is cited, in accordance with accepted academic practice. No use, distribution or reproduction is permitted which does not comply with these terms. 